\title{
Adult acute respiratory distress syndrome
}

INSERM

\section{Source}

INSERM. (1999). Orphanet: an online rare disease and orphan drug data base. Adult acute respiratory distress syndrome. ORPHA:70578

Adult acute respiratory distress syndrome is a very severe form of acute pulmonary failure secondary to capillary permeability impairment. The symptoms include dyspnea, hypotension and multovisceral failure. The disease is characterized by bilateral pulmonary infiltrates and severe hypoxemia due to increased alveolar-capillary permeability. The severity depends on the degree of alveolar epithelial injury, with a mortality rate of 30$50 \%$. 